PROCEEDINGS OF THE AMERICAN MATHEMATICAL SOCIETY

Volume 127, Number 4, April 1999, Pages 1065-1074

S 0002-9939(99)04590-6

\title{
EQUILIBRIUM PROBLEMS ASSOCIATED WITH FAST DECREASING POLYNOMIALS
}

\author{
A. B. J. KUIJLAARS AND P. D. DRAGNEV
}

(Communicated by J. Marshall Ash)

\begin{abstract}
The determination of the support of the equilibrium measure in the presence of an external field is important in the theory of weighted polynomials on the real line. Here we present a general condition guaranteeing that the support consists of at most two intervals. Applying this to the external fields associated with fast decreasing polynomials, we extend previous results of Totik and Kuijlaars-Van Assche. In the proof we use the iterated balayage algorithm which was first studied by Dragnev.
\end{abstract}

\section{Introduction AND STATEMENT OF RESUlts}

Let $w(x)=\exp (-Q(x))$ be a positive continuous weight defined on a compact set $\Sigma \subset \mathbb{R}$. The study of weighted polynomials $w^{n} P_{n}$, $\operatorname{deg} P_{n} \leq n$, has found important applications in the theory of orthogonal polynomials on the real line; see e.g. [4], [10], [12], [17]. See also [14] where other applications can be found as well.

In this theory a fundamental role is played by a probability measure $\mu_{w}$ with compact support $S_{w} \subset \Sigma$ characterized by the equations

$$
\begin{array}{ll}
U^{\mu_{w}}(x)+Q(x)=F_{w}, & \text { q.e. on } S_{w}, \\
U^{\mu_{w}}(x)+Q(x) \geq F_{w}, & \text { q.e. on } \Sigma .
\end{array}
$$

Here $F_{w}$ is a uniquely determined constant, $U^{\mu_{w}}$ denotes the logarithmic potential of $\mu_{w}$, i.e.,

$$
U^{\mu_{w}}(z)=\int \log \frac{1}{|z-t|} d \mu_{w}(t), \quad z \in \mathbb{C},
$$

and q.e. means quasi-everywhere, i.e., except for a set of logarithmic capacity zero. The measure $\mu_{w}$ is called the equilibrium measure (or extremal measure) associated with the external field $Q$. Its support $S_{w}$ is called the extremal support. See [8] for an interpretation of (1.1) as a contact problem in elasticity theory.

A complicating factor in solving (1.1) is that the support $S_{w}$ is not known in advance. It is part of the problem to determine the nature of $S_{w}$. The simplest

Received by the editors December 12, 1996 and, in revised form, July 16, 1997.

1991 Mathematics Subject Classification. Primary 41A10, 31A15.

Key words and phrases. Equilibrium measures, extremal support, balayage, fast decreasing polynomials.

The first author is supported by a postdoctoral fellowship of the Belgian National Fund for Scientific Research, Scientific Research Network nr WO.011.96N: Fundamental Methods and Techniques in Mathematics. The research of the second author is in partial fulfillment of the Ph.D. requirements at the University of South Florida. 
case is that $S_{w}$ is an interval, since then there are explicit formulas available for the measure $\mu_{w}$. This holds for example, if $\Sigma$ is an interval and the external field $Q$ is convex, which is the case that is studied most in the literature.

Recently, external fields of the form

$$
Q(x)=-c x^{\alpha}, \quad x \in[0,1],
$$

were found to be of interest in connection with the construction of fast decreasing polynomials. In this context, a sequence of polynomials $\left\{P_{n}\right\}_{n=0}^{\infty}$, $\operatorname{deg} P_{n} \leq n$, is fast decreasing if for some $M>0$, we have

$$
P_{n}(0)=1, \quad\left|P_{n}(x)\right| \leq M \exp \left(-c n x^{\alpha}\right), \quad x \in[0,1] .
$$

The right-hand side gives a prescribed rate of decrease of the polynomials on the interval $[0,1]$. The existence of polynomials satisfying (1.3) depending on the parameters $\alpha$ and $c$ was investigated in a number of papers [6], [7], [16]. The results may be summarized as follows. Let $w(x)=\exp \left(c x^{\alpha}\right)$ on $[0,1]$ and let $\mu_{w}$ be the equilibrium measure associated with the external field (1.2). Then fast decreasing polynomials (1.3) exist if and only if equality holds in (1.1) for $x=0$, i.e., $U^{\mu_{w}}(0)=F_{w}$. In particular, a sufficient condition is that $0 \in S_{w}$. Therefore, the determination of the support of $\mu_{w}$ is of importance here.

The case $\alpha \leq 1$ was studied by Lubinsky and Totik [11] and Totik [16], [17], who found that polynomials satisfying (1.3) exist if and only if

$$
c \leq c_{0}(\alpha):=\frac{2 \sqrt{\pi} \Gamma(\alpha)}{\Gamma(\alpha-1 / 2)}, \quad 1 / 2<\alpha \leq 1,
$$

while for $\alpha \leq 1 / 2$, fast decreasing polynomials satisfying (1.3) do not exist if $c>0$. For $\alpha \leq 1$, the external field is convex and the extremal support is an interval containing 1 for every $c$. Thus the problem comes down to determining whether the support is $[0,1]$.

The case $\alpha>1$ is more complicated, since $Q$ is no longer convex and the extremal supports are not necessarily intervals. This case was studied by Kuijlaars and Van Assche [7], who determined the optimal constant $c_{0}(\alpha)$, thereby solving a problem posed in [18]. It is not given by (1.4) but by a more complicated formula; see [7] for details. What was proved is that for $c \geq c_{0}(\alpha)$, the extremal support is an interval of the form $[b, 1]$ with $b>0$. For $c=c_{0}(\alpha)$, the support does not contain 0 , but still the equality $U^{\mu_{w}}(0)=F_{w}$ holds.

What remained open is what the extremal support looks like for $c<c_{0}(\alpha)$ and it is one of the purposes of this paper to fill this gap. We prove that the extremal support can consist of at most two intervals.

Theorem 1. Let $\alpha>1$ and $c>0$. Let $w(x)=\exp (-Q(x))$ with $Q$ given by (1.2). Then there are positive constants $c_{1}(\alpha)<c_{0}(\alpha)$, depending only on $\alpha$, such that the following hold.

(1) If $c \leq c_{1}(\alpha)$, then $S_{w}=[0,1]$.

(2) If $c \in\left(c_{1}(\alpha), c_{0}(\alpha)\right)$, then $S_{w}$ has the form $[0, a] \cup[b, 1]$ with $0<a<b<1$.

(3) If $c \geq c_{0}(\alpha)$, then $S_{w}$ has the form $[b, 1]$ with $0<b<1$.

That the support cannot be worse than two intervals may come somewhat as a surprise, since numerical calculations of weighted Leja points seemed to indicate that several intervals could appear and it was even suggested that for some $\alpha$, chaos might appear [18]. Knowing the above result one has to conclude that the 
numerical calculations were not reliable. The calculation of a large number of Leja points seems to be a process which is very unstable.

Theorem 1 will follow from a result which gives general conditions guaranteeing that the extremal support consists of at most two intervals. To describe these conditions, we assume that $\Sigma=[a, b]$ and that $Q$ is differentiable with a Hölder continuous derivative, i.e., $Q \in C^{1+\epsilon}([a, b])$ for some $\epsilon>0$. Then it is well-known $[3],[13]$ that the singular integral equation

$$
\int_{a}^{b} \frac{v(t)}{x-t} d t=Q^{\prime}(x), \quad a<x<b
$$

has a unique solution satisfying

$$
\int_{a}^{b} v(t) d t=1
$$

Note that $v(t)$ is not necessarily $\geq 0$. But if it is, then it follows that $v(t) d t$ is the equilibrium measure with external field $Q$. The solution $v(t)$ is given by the explicit expression

$$
v(t)=\frac{1}{\pi \sqrt{(b-t)(t-a)}}\left[1+\frac{1}{\pi} \int_{a}^{b} \frac{Q^{\prime}(s)}{s-t} \sqrt{(b-s)(s-a)} d s\right], \quad a<t<b,
$$

where the integral is again a Cauchy principal value integral, see [3, p. 428].

Then we have the following result.

Theorem 2. Suppose $w=\exp (-Q)$ with $Q \in C^{1+\epsilon}([a, b])$ for some $\epsilon>0$ and let $v(t)$ be given by (1.7).

(1) If $\pi \sqrt{(b-t)(t-a)} v(t)$ is increasing on $[a, b]$, then the extremal support $S_{w}$ is an interval containing $b$.

(2) If $\pi \sqrt{(b-t)(t-a)} v(t)$ is decreasing on $[a, b]$, then the extremal support is an interval containing $a$.

(3) If there is a $t_{0} \in(a, b)$ such that $\pi \sqrt{(b-t)(t-a)} v(t)$ is decreasing on $\left[a, t_{0}\right]$ and increasing on $\left[t_{0}, b\right]$, then the extremal support is either an interval containing $a$, or an interval containing $b$, or the union of an interval containing $a$ with an interval containing $b$.

We note that also parts (1) and (2) have not appeared in the literature before.

The proof of Theorem 2 is based on an algorithmic approach to solve the equilibrium problem (1.1). This so-called iterated balayage algorithm was first studied by Dragnev [1] in his thesis. It is described in Section 2 and the properties that are needed for Theorem 2 are proved. In Section 3 we give the proof of Theorem 2 and in Section 4 we prove Theorem 1 by showing that for the external field $Q$ from (1.2), the condition of Theorem 2(1) is satisfied.

Throughout the paper we use $\sigma^{+}$and $\sigma^{-}$to denote the positive and negative parts, respectively, in the Jordan decomposition of a signed measure $\sigma=\sigma^{+}-\sigma^{-}$.

\section{The ITERATED BALAYAGE ALGORITHM}

We start with a lemma.

Lemma 3. Suppose $w(x)=\exp (-Q(x)), x \in \Sigma$, and

$$
Q(x)+U^{\sigma}(x)=C, \quad \text { for q.e. } x \in \Sigma
$$


where $C$ is a constant and $\sigma$ is a signed measure on $\Sigma$ such that $\int d \sigma=1$. Suppose $U^{\sigma^{+}}$is continuous and $U^{\sigma^{-}}$is bounded on $\Sigma$. Then

$$
\mu_{w} \leq \sigma^{+} \quad \text { and } \quad S_{w} \subset \operatorname{supp}\left(\sigma^{+}\right) .
$$

Proof. From (1.1) and (2.1) it follows that

$$
\begin{array}{ll}
U^{\sigma^{+}}(x)=U^{\mu_{w}+\sigma^{-}}(x)+C-F_{w}, & \text { q.e. on } S_{w}, \\
U^{\sigma^{+}}(x) \leq U^{\mu_{w}+\sigma^{-}}(x)+C-F_{w}, & \text { q.e. on } \Sigma .
\end{array}
$$

Since $\int d \sigma=1$, we have $\left\|\sigma^{+}\right\|-\left\|\sigma^{-}\right\|=1$, so that $\left\|\sigma^{+}\right\|=\left\|\mu_{w}+\sigma^{-}\right\|$. Therefore we can apply the principle of domination for logarithmic potentials (see e.g. [14], [15]), and we obtain that the inequality $(2.3)$ holds for every $x \in \mathbb{C}$. Then by a theorem of de La Vallée Poussin (see [14] or [17]), we have $\left.\left(\mu_{w}+\sigma^{-}\right)\right|_{S_{w}} \leq\left.\left(\sigma^{+}\right)\right|_{S_{w}}$. Hence $\mu_{w} \leq \sigma^{+}$and the lemma follows.

The lemma is closely related to Theorem 2.6 of [2].

Lemma 3 provides the basis for the iterated balayage algorithm. We recall the notion of balayage onto a compact set; see [9], [14], [15], [17]. If $K$ is a compact subset of the real line with positive logarithmic capacity and $\nu$ is any finite positive measure on $\mathbb{C}$ with compact support, then there is a unique positive measure $\hat{\nu}$ supported on $K$ such that $\|\hat{\nu}\|=\|\nu\|, \hat{\nu}$ vanishes on the irregular points of $K$ and for some constant $C$,

$$
U^{\hat{\nu}}(z)=U^{\nu}(z)+C, \quad \text { q.e. on } K .
$$

The measure $\hat{\nu}$ is called the balayage of $\nu$ onto $K$ and we denote it by $\operatorname{Bal}(\nu ; K)$. For a signed measure $\nu$, we define $\operatorname{Bal}(\nu ; K):=\operatorname{Bal}\left(\nu^{+} ; K\right)-\operatorname{Bal}\left(\nu^{-} ; K\right)$.

We introduce an operator $J$ defined on a subset of the space of finite signed measures with compact support in $\mathbb{R}$. The domain of $J$ consists of all signed measures $\sigma$ with $\int d \sigma=1$ having the property that $\operatorname{cap}\left(\operatorname{supp}\left(\sigma^{+}\right)\right)>0$. For such $\sigma$, we define

$$
J(\sigma):=\sigma^{+}-\operatorname{Bal}\left(\sigma^{-} ; \operatorname{supp}\left(\sigma^{+}\right)\right)=\operatorname{Bal}\left(\sigma ; \operatorname{supp}\left(\sigma^{+}\right)\right) .
$$

Thus the operator $J$ sweeps the negative part of the measure $\sigma$ onto the support of the positive part, so that in particular $J(\sigma)^{+} \leq \sigma^{+}$. For each $k \geq 1, J^{k}(\sigma)$ denotes the $k$ th iterate of $\sigma$ under $J$, if it is defined.

Suppose we are in the situation of Lemma 3. Then it follows from (2.2) that $\operatorname{cap}\left(\operatorname{supp}\left(\sigma^{+}\right)\right) \geq \operatorname{cap}\left(S_{w}\right)>0$, so that $\sigma_{1}:=J(\sigma)$ is defined. Put $\Sigma_{1}:=\operatorname{supp}\left(\sigma^{+}\right)$. Then we have by (2.1) and the property (2.4) of balayage that

$$
Q(x)+U^{\sigma_{1}}(x)=C_{1}, \quad \text { for q.e. } x \in \Sigma_{1} .
$$

It can be shown that Lemma 3 applies again so that $\mu_{w} \leq \sigma_{1}^{+} \leq \sigma^{+}$. Continuing in this way, we obtain that $\sigma_{k}:=J^{k}(\sigma)$ exists for every $k$, and that

$$
S_{w} \subset \operatorname{supp}\left(\sigma_{k}^{+}\right) \quad \text { and } \quad \sigma_{k}^{+} \geq \sigma_{k+1}^{+} \geq \cdots \geq \mu_{w} .
$$

It seems reasonable to expect from $(2.6)$ that the sequence $\left\{\sigma_{k}^{+}\right\}$converges to $\mu_{w}$ and indeed, this can be proved under quite general assumptions (see [1]). It is not known if the algorithm converges to $\mu_{w}$ in the full generality presented here. However, for the proof of Theorem 2 we need it only in a very special case where the convergence can be easily shown. This will be done in the next section. 


\section{Proof of Theorem 2}

We need one more lemma about balayage.

Lemma 4. Let $K$ be a compact subset of $\mathbb{R}$. Let $\nu$ be a positive measure with compact support with $\nu(K)=0$, and let $\hat{\nu}$ be the balayage of $\nu$ onto $K$.

(1) Suppose $K=[a, b]$. Then $\hat{\nu}$ is absolutely continuous with respect to Lebesgue measure. If $\operatorname{supp}(\nu) \subset(-\infty, a]$, then $\pi \sqrt{(b-t)(t-a)} d \hat{\nu} / d t$ is decreasing on $[a, b]$. If $\operatorname{supp}(\nu) \subset[b, \infty)$, then $\pi \sqrt{(b-t)(t-a)} d \hat{\nu} / d t$ is increasing on $[a, b]$.

(2) Suppose $K=[a, b] \cup[c, d]$ with $a<b<c<d$. If $\operatorname{supp}(\nu) \subset[b, c]$, then $\hat{\nu}$ is absolutely continuous and $\pi \sqrt{(d-t)(t-a)} d \hat{\nu} / d t$ is increasing on $[a, b]$ and decreasing on $[c, d]$.

Proof. (1) This is immediate from the explicit representation

$$
\pi \sqrt{(b-t)(t-a)} \frac{d \hat{\nu}}{d t}=\int \frac{\sqrt{(b-s)(a-s)}}{|s-t|} d \nu(s), \quad a<t<b ;
$$

see e.g. [14, Section II.4].

(2) It is enough to prove (2) for $\nu=\delta_{s}$, the point mass of a point $s$ where $b<s<c$, since for general $\nu$ we have

$$
\frac{d \hat{\nu}}{d t}=\int_{b}^{c} \frac{d \hat{\delta}_{s}}{d t} d \nu(s)
$$

Without loss of generality we may further assume that $s=0$. We note that the usual equilibrium measure (with external field $Q \equiv 0$ ) of the set $[1 / b, 1 / a] \cup[1 / d, 1 / c]$ has a density given by

$$
u(t):=\frac{|t-X|}{\pi \sqrt{|t-1 / a||t-1 / b||t-1 / c||t-1 / d|}}, \quad t \in[1 / b, 1 / a] \cup[1 / d, 1 / c],
$$

with $X$ some point in the interval $(1 / a, 1 / d)$; see [15, Lemma 4.4.1]. This means that for some constant $C_{1}$,

$$
-\int \log |x-t| u(t) d t=C_{1}, \quad x \in[1 / b, 1 / a] \cup[1 / d, 1 / c] .
$$

If we change variables $t \mapsto 1 / t$ and write $1 / x$ instead of $x$, we obtain after simple calculations

$$
-\int \log |x-t| \frac{u(1 / t)}{t^{2}} d t=-\log |x|+C_{2}, \quad x \in[a, b] \cup[c, d],
$$

with a (different) constant $C_{2}$. Thus

$$
\frac{d \hat{\delta}_{0}}{d t}=\frac{u(1 / t)}{t^{2}}=\frac{|1-t X|}{|t|} \frac{\sqrt{a b c d}}{\pi \sqrt{|t-a||t-b||t-c||t-d|}}, \quad t \in[a, b] \cup[c, d] .
$$

Then (2) easily follows, since $X \in(1 / a, 1 / d)$.

Lemma 5. (1) Let $a<b$ and $v \in C((a, b))$ such that $\int_{a}^{b} v(t) d t=1$. Put $d \sigma(t)=v(t) d t$. Suppose $\pi \sqrt{(b-t)(t-a)} v(t)$ is increasing on $[a, b]$. Then $\operatorname{supp}\left(\sigma^{+}\right)=\left[a^{*}, b\right]$ for some $a^{*} \in[a, b)$, and $J(\sigma)$ exists and has density $v^{*}$ on $\left[a^{*}, b\right]$ such that $\pi \sqrt{(b-t)\left(t-a^{*}\right)} v^{*}(t)$ is increasing on $\left[a^{*}, b\right]$. 
(2) Let $a<b$ and $v \in C((a, b))$ such that $\int_{a}^{b} v(t) d t=1$. Put $d \sigma(t)=v(t) d t$. Suppose $\pi \sqrt{(b-t)(t-a)} v(t)$ is decreasing on $[a, b]$. Then $\operatorname{supp}\left(\sigma^{+}\right)=\left[a, b^{*}\right]$ for some $b^{*} \in(a, b]$, and $J(\sigma)$ exists and has density $v^{*}$ on $\left[a, b^{*}\right]$ such that $\pi \sqrt{\left(b^{*}-t\right)(t-a)} v^{*}(t)$ is increasing on $\left[a, b^{*}\right]$.

(3) Let $a<b \leq c<d$ and $v \in C((a, b) \cup(c, d))$ with $\int_{a}^{b} v(t) d t+\int_{c}^{d} v(t) d t=1$. Put $d \sigma(t)=v(t) d t$. Suppose $\pi \sqrt{(d-t)(t-a)} v(t)$ is decreasing on $[a, b]$ and increasing on $[c, d]$. Then there are 3 possibilities:

(a) $\operatorname{supp}\left(\sigma^{+}\right)=\left[a, b^{*}\right]$ for some $b^{*} \in(a, b]$. Then $J(\sigma)$ exists and has a continuous density $v^{*}$ on $\left(a, b^{*}\right)$ such that $\pi \sqrt{\left(b^{*}-t\right)(t-a)} v^{*}(t)$ is decreasing on $\left[a, b^{*}\right]$.

(b) $\operatorname{supp}\left(\sigma^{+}\right)=\left[c^{*}, d\right]$ for some $c^{*} \in[c, d)$. Then $J(\sigma)$ exists and has a continuous density $v^{*}$ on $\left(c^{*}, d\right)$ such that $\pi \sqrt{(d-t)\left(t-c^{*}\right)} v^{*}(t)$ is increasing on $\left[c^{*}, d\right]$.

(c) $\operatorname{supp}\left(\sigma^{+}\right)=\left[a, b^{*}\right] \cup\left[c^{*}, d\right]$ for some $b^{*} \in(a, b]$ and $c^{*} \in[c, d)$. Then $J(\sigma)$ exists and has a continuous density $v^{*}$ on $\left(a, b^{*}\right) \cup\left(c^{*}, d\right)$ such that $\pi \sqrt{(d-t)(t-a)} v^{*}(t)$ is decreasing on $\left[a, b^{*}\right]$ and increasing on $\left[c^{*}, d\right]$.

Proof. (1) Since $\int d \sigma=1$, it is clear that $\operatorname{supp}\left(\sigma^{+}\right)$has the form $\left[a^{*}, b\right]$ with $a^{*}<b$. Thus cap $\left(\operatorname{supp}\left(\sigma^{+}\right)\right)>0$, so that $J(\sigma)$ exists. This measure has a continuous density

$$
v^{*}(t)=v(t)-\frac{d \hat{\sigma}^{-}}{d t}(t), \quad t \in\left(a^{*}, b\right),
$$

where $\hat{\sigma}^{-}$is the balayage of $\sigma^{-}$onto $\operatorname{supp}\left(\sigma^{+}\right)$. From Lemma 4(a) we see that $\pi \sqrt{(b-t)\left(t-a^{*}\right)} d \hat{\sigma}^{-} / d t$ decreases on $\left[a^{*}, b\right]$, while we get from the assumptions that $\pi \sqrt{(b-t)\left(t-a^{*}\right)} v(t)$ increases on $\left[a^{*}, b\right]$. This proves (1).

(2) The proof of part (2) is analogous.

(3) It is clear that $\operatorname{supp}\left(\sigma^{+}\right)$has one of the forms (a), (b) or (c) and that in all cases $J(\sigma)$ exists and has a continuous density

$$
v^{*}(t)=v(t)-\frac{d \hat{\sigma}^{-}}{d t}(t), \quad t \in \operatorname{int}\left(\operatorname{supp}\left(\sigma^{+}\right)\right),
$$

where $\hat{\sigma}^{-}$is the balayage of $\sigma^{-}$onto $\operatorname{supp}\left(\sigma^{+}\right)$. Using Lemma 4(1) in cases (a) and (b) and Lemma 4(2) in case (c), we obtain (3) in the same way as we obtained part (1).

Having Lemma 5 we are ready for the proof of Theorem 2.

Proof of Theorem 2. (1) Let $d \sigma_{0}(t):=v(t) d t$ and put $\sigma_{k}:=J^{k}\left(\sigma_{0}\right), k \geq 1$. Using Lemma 5(1) repeatedly, we see that these measures exist. It is also clear from Lemma 5(1) that for some non-decreasing sequence $\left\{a_{k}\right\}_{k=0}^{\infty}$, we have $\operatorname{supp}\left(\sigma_{k-1}^{+}\right)=$ $\left[a_{k}, b\right]$, so that $\sigma_{k}$ is the balayage of $\sigma_{0}$ onto $\left[a_{k}, b\right]$, and that $\pi \sqrt{(b-t)\left(t-a_{k}\right)} v_{k}(t)$ increases on $\left[a_{k}, b\right]$, where $v_{k}$ is the density of $\sigma_{k}$. Now let

$$
a_{\infty}:=\lim _{k \rightarrow \infty} a_{k}
$$

Since $\mu_{w} \leq \sigma_{k}^{+}$for every $k$, see (2.6), it follows that $\operatorname{supp}\left(\mu_{w}\right) \subset\left[a_{\infty}, b\right]$ and therefore $a_{\infty}<b$. Let $\sigma_{\infty}$ denote the balayage of $\sigma_{0}$ onto $\left[a_{\infty}, b\right]$. Since $\sigma_{k}$ is the balayage onto $\left[a_{k}, b\right]$, we have

$$
\lim _{k \rightarrow \infty} \sigma_{k}=\sigma_{\infty}
$$


where the convergence is in weak* sense. $\operatorname{Since} \operatorname{supp}\left(\sigma_{k}^{-}\right)=\left[a_{k}, a_{k+1}\right]$ and the total masses $\left\|\sigma_{k}^{-}\right\|$are decreasing (because of (2.6) and the fact that $\left\|\sigma_{k}^{+}\right\|=1+\left\|\sigma_{k}^{-}\right\|$), it follows that

$$
\lim _{k \rightarrow \infty} \sigma_{k}^{-}=c \delta_{a_{\infty}}
$$

with $c \geq 0$. Next we note that $v_{k+1} \leq v_{k} \leq v$ on $\left[a_{k+1}, b\right]$. This readily implies that

$$
\lim _{k \rightarrow \infty} \sigma_{k}^{+}=v_{\infty}(t) d t, \quad a_{\infty}<t<b,
$$

with $v_{\infty}(t):=\lim _{k} v_{k}(t), a_{\infty}<t<b$. Then by (3.1), (3.2), (3.3), we have that $\sigma_{\infty}=v_{\infty}(t) d t-c \delta_{a_{\infty}}$. This implies that $c=0$ since $\sigma_{\infty}$ has no point masses, being the balayage onto $\left[a_{\infty}, b\right]$. Consequently, $\sigma_{\infty}$ is a non-negative measure and it follows that $\sigma_{\infty}=\mu_{w}$. Since $\pi \sqrt{(b-t)\left(t-a_{\infty}\right)} v_{\infty}(t)$ is increasing on $\left[a_{\infty}, b\right]$, it follows that $S_{w}$ is an interval containing $b$. It could be strictly smaller than $\left[a_{\infty}, b\right]$ if $v_{\infty}$ vanishes on some interval containing $a_{\infty}$. This proves part (1) of the theorem.

(2) The proof of (2) is analogous to the proof of part (1).

(3) We closely follow the proof of part (1). We put $d \sigma_{0}(t):=v(t) d t$ and $\sigma_{k}:=$ $J^{k}\left(\sigma_{0}\right), k \geq 1$. Also put $a_{0}=a, b_{0}=c_{0}=t_{0}$ and $d_{0}=b$.

Using Lemma $5(3)$ (c) repeatedly, we find that there is an $N \geq 0$, a non-increasing sequence $\left\{b_{k}\right\}_{k=0}^{N}$ and a non-decreasing sequence $\left\{c_{k}\right\}_{k=0}^{N}$ such that

$$
\operatorname{supp}\left(\sigma_{k-1}^{+}\right)=\left[a_{0}, b_{k}\right] \cup\left[c_{k}, d_{0}\right], \quad k \geq 1,
$$

and $\pi \sqrt{\left(d_{0}-t\right)\left(t-a_{0}\right)} d \sigma_{k} / d t(t)$ decreases on $\left[a_{0}, b_{k}\right]$ and increases on $\left[c_{k}, d_{0}\right]$. The number $N$ may be finite or infinite, depending on whether Lemma 5(3)(c) applies a finite or infinite number of times. If it is finite, then we have that cases (a) or (b) of Lemma 5(3) apply to $\sigma_{N}$ and we are back to parts (1) or (2). It then follows from what has been proven there that $S_{w}$ is an interval containing $a$ or an interval containing $b$.

If $N$ is infinite, we put

$$
b_{\infty}:=\lim _{k \rightarrow \infty} b_{k}, \quad c_{\infty}:=\lim _{k \rightarrow \infty} c_{k}
$$

and we have that $\sigma_{k}$ is the balayage of $\sigma_{0}$ onto $\left[a_{0}, b_{k}\right] \cup\left[c_{k}, d_{0}\right]$. Then

$$
\lim _{k \rightarrow \infty} \sigma_{k}=\sigma_{\infty}
$$

where $\sigma_{\infty}$ is the balayage of $\sigma_{0}$ onto $\left[a_{0}, b_{\infty}\right] \cup\left[c_{\infty}, d_{0}\right]$. Now, just as in the proof of part (1) it follows that $\mu_{w}=\sigma_{\infty}$ with a density $v_{\infty}$ given by

$$
v_{\infty}(t):=\lim _{k \rightarrow \infty} \frac{d \sigma_{k}}{d t}(t), \quad t \in\left(a_{0}, b_{\infty}\right) \cup\left(c_{\infty}, d_{0}\right) .
$$

Since $\pi \sqrt{\left(d_{0}-t\right)\left(t-a_{0}\right)} v_{\infty}(t)$ decreases on $\left[a_{0}, b_{\infty}\right]$ and increases on $\left[c_{\infty}, d_{0}\right]$, it follows that $S_{w}$ is either an interval containing $a_{0}$ or an interval containing $d_{0}$ or consists of two intervals. It is, e.g., an interval containing $a_{0}$ if $c_{\infty}=d_{0}$ or if $v_{\infty}$ vanishes on $\left(c_{\infty}, d_{0}\right)$.

This completes the proof of Theorem 2.

\section{Proof of Theorem 1}

For $\alpha>1$, we define

$$
G_{\alpha}(t):=-\frac{1}{\pi} \int_{0}^{1} \frac{s^{\alpha-1}}{s-t} \sqrt{s(1-s)} d s, \quad 0 \leq t \leq 1,
$$


where the integral is a principal value integral if $0<t<1$. For the proof of Theorem 1 we need the following property of the function $G_{\alpha}$.

Lemma 6. Let $\alpha>1$. There is a $t_{0} \in(0,1)$ such that $G_{\alpha}$ decreases on $\left[0, t_{0}\right]$ and increases on $\left[t_{0}, 1\right]$.

Proof. Put $k:=[\alpha]+1$, where $[\alpha]$ denotes the integer part of $\alpha$. The lemma follows from the following three properties of $G_{\alpha}$.

(1) $G_{\alpha}^{(l)}(0)<0$ for all $l=0,1, \ldots, k-1$.

(2) $G_{\alpha}(1)>0$.

(3) $G_{\alpha}^{(k)}(t) \geq 0$ for $0<t<1$.

Indeed, it follows from (3) that $G_{\alpha}^{(k-1)}$ is increasing on $[0,1]$ and therefore it has at most one zero. If there were no zero, then we first get from (1) that $G_{\alpha}^{(k-1)}<0$ on $(0,1)$ and then it would follow from $(1)$ that $G_{\alpha}<0$ on $(0,1)$, which is impossible because of (2). Thus $G_{\alpha}^{(k-1)}$ has exactly one zero and so $G_{\alpha}^{(k-2)}$ decreases until it reaches a minimum and then increases. By (1) with $l=k-2, G_{\alpha}^{(k-2)}$ has at most one zero and in the same way as before it has exactly one zero. Continuing in this way, we obtain that $G_{\alpha}^{\prime}$ has exactly one zero $t_{0} \in(0,1)$ and the lemma follows.

So what remains is to prove (1), (2) and (3).

(1) For $l \leq k-1$, it is easy to obtain from (4.1) that

$$
G_{\alpha}^{(l)}(0)=-\frac{l !}{\pi} \int_{0}^{1} s^{\alpha-l-3 / 2}(1-s)^{1 / 2} d s<0,
$$

which is (1). Here it may be noted that $G_{\alpha}^{(l)}(0)=-\infty$ if $l \geq \alpha-1 / 2$.

(2) follows immediately from (4.1).

(3) Put $\phi(x):=x^{\alpha+1}-x^{\alpha}$. From (4.1) we note that

$$
G_{\alpha}(t)=\frac{1}{\pi} \int_{0}^{1} \frac{\phi(s)}{s-t} \frac{d s}{\sqrt{s(1-s)}}=\frac{1}{\pi} \int_{0}^{1} \frac{\phi(t)-\phi(s)}{t-s} \frac{d s}{\sqrt{s(1-s)}} .
$$

The Taylor expansion of $\phi$ with remainder term gives

$$
\frac{\phi(t)-\phi(s)}{t-s}=\sum_{j=1}^{k} \frac{1}{j !}(t-s)^{j-1} \phi^{(j)}(s)+\frac{1}{k !} \int_{s}^{t} \frac{(t-x)^{k}}{t-s} \phi^{(k+1)}(x) d x .
$$

The $k$ th derivative of the polynomial part vanishes. Thus

$$
\begin{aligned}
\left(\frac{d}{d t}\right)^{k}\left(\frac{\phi(t)-\phi(s)}{t-s}\right) & =\frac{1}{k !} \int_{s}^{t}\left(\frac{d}{d t}\right)^{k}\left(\frac{(t-x)^{k}}{t-s}\right) \phi^{(k+1)}(x) d x \\
& =\int_{s}^{t} \frac{(x-s)^{k}}{(t-s)^{k+1}} \phi^{(k+1)}(x) d x .
\end{aligned}
$$

Observe that $\phi^{(k+1)}(x) \geq 0$ by the choice of $k$. Thus (4.3) is non-negative for all $s, t \in(0,1)$ and then (3) follows from this and (4.2).

Remark. The function $G_{\alpha}$ can be expressed in terms of the Gauss hypergeometric function as

$$
G_{\alpha}(t)=\frac{\Gamma(\alpha+1 / 2)}{\sqrt{\pi} \Gamma(\alpha+1)} F(-\alpha, 1 ; 1 / 2 ; 1-t) ;
$$

see formula 3.228.3 in [5]. The proof of Lemma 6 can also be based on this formula. We thank Walter Van Assche for this remark. 
Proof of Theorem 1. Let $v(t), 0<t<1$, be given by (1.7) with $a=0$ and $b=1$, so that

$$
v(t)=\frac{1}{\pi \sqrt{t(1-t)}}\left[1+\alpha c G_{\alpha}(t)\right], \quad 0<t<1,
$$

with $G_{\alpha}$ given by (4.1). Then by Lemma 6 and (4.4), $\pi \sqrt{t(1-t)} v(t)$ is decreasing on $\left[0, t_{0}\right]$ and increasing on $\left[t_{0}, 1\right]$. It follows from Theorem $2(3)$ that $S_{w}$ is either an interval containing 0 , or an interval containing 1 , or the union of an interval containing 0 and an interval containing 1.

If $v$ is non-negative on $[0,1]$, then $S_{w}=[0,1]$ and this is the case if and only if $c \leq c_{1}(\alpha)$ with

$$
c_{1}(\alpha):=-\frac{1}{\alpha G_{\alpha}\left(t_{0}\right)}
$$

which is a positive number. Hence we have (1).

It was proved in [7, Theorem 4] that there exists a constant $c_{0}(\alpha)$ such that $S_{w}$ is an interval containing 1 for $c \geq c_{0}(\alpha)$ and $0 \in S_{w}$ for $c<c_{0}(\alpha)$. This gives (3).

In the remaining case $c \in\left(c_{1}(\alpha), c_{0}(\alpha)\right)$ the support $S_{w}$ has to consist of two intervals, one containing 0 and the other containing 1 . This proves (2) and we have completed the proof of the theorem.

\section{REFERENCES}

[1] P.D. Dragnev, Constrained energy problems for logarithmic potentials, Ph.D. Thesis, University of South Florida, Tampa, FL, 1997.

[2] P.D. Dragnev and E.B. Saff, Constrained energy problems with applications to orthogonal polynomials of a discrete variable, J. Anal. Math. 72 (1997), 223-259. CMP 98:04

[3] F.D. Gakhov, Boundary Value Problems, Pergamon Press, Oxford, 1966. MR 33:6311

[4] A.A. Gonchar and E.A. Rakhmanov, Equilibrium measure and the distribution of zeros of extremal polynomials, Mat. Sb. 125 (1984), 117-127 (Russian). English transl. in Math. USSR Sb. 53 (1986), 119-130. MR 86f:41002

[5] I.S. Gradshteyn and I.M. Ryzhik, Table of Integrals, Series, and Products, Academic Press, Orlando, 1980. MR 81g:33001

[6] K.G. Ivanov and V. Totik, Fast decreasing polynomials, Constr. Approx. 6 (1990), 1-20. MR 90k:26023

[7] A.B.J. Kuijlaars and W. Van Assche, A problem of Totik on fast decreasing polynomials, Constr. Approx. 14 (1998), 97-112. CMP 98:05

[8] - A contact problem in elasticity related to weighted polynomials on the real line, Rend. Circ. Mat. Palermo Suppl. 52 (1998), 575-587.

[9] N.S. Landkof, Foundations of Modern Potential Theory, Springer-Verlag, New York, 1972. MR 50:2520

[10] D.S. Lubinsky and E.B. Saff, Strong Asymptotics for Extremal Polynomials Associated with Weights on $\mathbb{R}$, Lecture Notes Math., vol. 1305, Springer-Verlag, New York, 1988. MR 89m:41013

[11] D.S. Lubinsky and V. Totik, How to discretize a logarithmic potential?, Acta Sci. Math. (Szeged) $\mathbf{5 7}$ (1993), 419-428.

[12] H.N. Mhaskar and E.B. Saff, Extremal problems for polynomials with exponential weights, Trans. Amer. Math. Soc. 285 (1984), 203-234. MR 86b:41024

[13] N.I. Muskhelishvili, Singular Integral Equations, Noordhoff, Groningen, 1953. MR 15:434e

[14] E.B. Saff and V. Totik, Logarithmic Potentials with External Fields, Springer-Verlag, Berlin, 1997.

[15] H. Stahl and V. Totik, General Orthogonal Polynomials, Cambridge University Press, Cambridge, 1992.

[16] V. Totik, Fast decreasing polynomials via potentials, J. Anal. Math. 62 (1994), 131-154. MR 96e: 41010 
[17] - Weighted Approximation with Varying Weight, Lecture Notes Math., vol. 1569, Springer-Verlag, Berlin, 1994. MR 96f:41002

[18] _ Problem 13.5, in: Linear and Complex Analysis Problem Book 3, Part II (V.P. Havin and N.K. Nikolski, eds.), Lecture Notes Math., vol. 1574, Springer-Verlag, Berlin, 1994.

Department of Mathematics, Katholieke Universiteit Leuven, Celestijnenlaan 200 B, B-3001 Leuven, Belgium

E-mail address: arno@wis.kuleuven.ac.be

Department of Mathematics, University of South Florida, Tampa, Florida 33620

Current address: Department of Mathematical Sciences, Indiana University Purdue University Fort Wayne, Fort Wayne, Indiana 46805

E-mail address: dragnevp@ipfw.edu 